\title{
Avaliação do emprego da haste femoral curta na fratura trocantérica instável do fêmur*
}

\author{
Evaluating the use of a proximal femoral nail \\ in unstable trochanteric fracture of the femur
}

João Antonio Matheus Guimarães ${ }^{1}$, Ana Carolina Abdon Guimarães², José Sérgio Franco ${ }^{3}$

\section{RESUMO}

Objetivo: Identificar a taxa de consolidação da fratura trocantérica instável submetida a osteossíntese com haste femoral curta $\left(\mathrm{PFN}^{\circledR}-\mathrm{AO} / \mathrm{ASIF}\right)$, em pacientes operados entre novembro de 1999 e março de 2004. Métodos: Foram analisados 45 pacientes com idades entre 60 e 93 anos, portadores de fratura trocantérica instável do fêmur, submetidos à osteossíntese com $\mathrm{PFN}^{\circledR}$ curto, mediante redução indireta em mesa de tração e auxílio de radioscopia. As fraturas foram classificadas de acordo com a classificação AO/OTA. A qualidade óssea foi avaliada através do índice de Singh, na rotina radiográfica pré-operatória. A qualidade da redução obtida e o posicionamento do implante foram avaliados pela radiografia pós-operatória em ânteroposterior e perfil do fêmur proximal, com análise

* Trabalho realizado no Instituto Nacional de Traumatologia e Ortopedia - Ministério da Saúde - INTO-MS - Rio de Janeiro (RJ), Brasil.

1. Mestre, Chefe da Divisão de Ortopedia e Traumatologia do Instituto Nacional de Traumatologia e Ortopedia - Ministério da Saúde - INTO-MS - Rio de Janeiro (RJ), Brasil.

2. Residente do 3o ano do Programa de Residência Médica do Instituto Nacional de Traumatologia e Ortopedia - Ministério da Saúde - INTO-MS - Rio de Janeiro (RJ), Brasil.

3. Doutor, Professor Associado e Chefe do Departamento de Ortopedia e Traumatologia da Universidade Federal do Rio de Janeiro - UFRJ - Rio de Janeiro (RJ), Brasil.

Endereço para correspondência: Rua Zacarias da Silva, 190, casa 2 - 22793-190 - Rio de Janeiro (RJ), Brasil. Fax: (21) 2294-8140.

E-mail: jomatheus@uol.com.br

Recebido em 6/5/08. Aprovado para publicação em 8/9/08.

Copyright RBO2008 do ângulo cervicodiafisário e a distância entre a ponta do parafuso deslizante e o centro da cabeça femoral, o chamado tip apex distance (TAD). Resultados: A média de idade dos pacientes estudados foi de 80,8 anos (60-93). O sexo feminino foi predominante, com 37 casos $(82,2 \%)$. O lado direito foi acometido em 22 casos $(48,89 \%)$ e o esquerdo em 23 $(51,1 \%)$. Todas as fraturas eram instáveis, sendo 22 do tipo $31 \mathrm{A2}(48,8 \%)$ e 23 do tipo $31 \mathrm{A3}(51,1 \%)$. O tempo médio de utilização da radioscopia foi de 102,4 segundos (61-185). A diferença entre o tempo de radioscopia para a redução e fixação dos dois grupos de fraturas estudados não foi significativa $(p=0,62)$. Com relação à qualidade óssea, $82,2 \%$ dos pacientes apresentavam índice de Singh menor que IV, caracterizando perda da arquitetura óssea normal. Quanto à consolidação da fratura, 44 casos evoluíram com êxito com tempo médio de 3,2 meses, variando entre dois e sete meses. Em sete casos ocorreu a necessidade de novo procedimento cirúrgico, sendo quatro para a retirada de material de síntese, devido a migração dos parafusos proximais da haste. Um caso de fratura do tipo 31A2, devido a uma redução inadequada em varo, ocorreu um cutout que necessitou de revisão cirúrgica. Outro caso de fratura do tipo $31 \mathrm{A2}$, evoluiu para necrose avascular da cabeça femoral, após consolidação da fratura. Por fim, um caso de fratura do tipo $31 \mathrm{A3}$, devido a redução inadequada no plano sagital, evoluiu para retarde de consolidação, com quebra da haste após $\mathbf{1 3}$ meses da cirurgia. Conclusão: A osteossíntese com haste proximal, como 
tratamento da fratura trocantérica instável do fêmur realizada em pacientes com idade superior a 60 anos, resultou na consolidação da maioria dos casos. As complicações foram distintas nos dois subgrupos estudados. A redução adequada da fratura antes da introdução da haste intramedular é fundamental para o sucesso do procedimento.

Descritores - Fraturas do quadril; Fixação interna de fraturas/métodos; Fraturas do femur; Pinos ortopédicos; Consolidação da fratura; Complicações pós-operatórias; Idoso

\section{ABSTRACT}

Objective: Identifying the rate of healing of unstable trochanteric fractures submitted to osteosynthesis with a proximal femoral nail (PFN ${ }^{\circledR}$ AO/ASIF), in patients operated on between November 1999 and March 2004. Methods: 45 patients were analyzed, ages ranging from 60 to 93, with unstable trochanteric fractures of the femur submitted to osteosynthesis with short $\mathrm{PFN}^{\circledR}$, with indirect reduction in a traction device guided by radioscopy. The fractures were classified according to the AO/OTA classification. Bone quality was evaluated by the Singh index in the post-operative radiographic routine. The quality of the reduction achieved and the positioning of the implant were evaluated by postoperative anteroposterior and profile $X$-ray of the proximal femur, with analysis of the cervicodiaphyseal angle and the distance between the tip of the sliding nail and the center of the femoral head, the so-called "tip apex distance" (TAD). Results: The mean age of patients studied was 80.8 years (60-93). Females prevailed, with 37 cases $(82.2 \%)$. The right side was involved in 22 cases (48.89\%) and the left side, in 23 (51.1\%). All fractures were unstable, 22 of them of the type $31 \mathrm{~A} 2$ (48.8\%), and 23, of the type 3143 (51.1\%). Mean time of use of radioscopy was 102.4 seconds (61-185). The difference in the radioscopy time for reduction and fixation between the two groups of fractures studied was not significant $(p=0.62)$. Regarding bone quality, $82.2 \%$ of the patients presented a Singh index lower than IV, characterizing loss of normal bone architecture. Regarding fracture healing, forty-four cases had a successful evolution in a mean time of 3.2 months, ranging from two to seven months. In seven cases, there was a need of a new surgery, four to remove the synthesis material due to migration of the proximal screws of the nail. One case of type $31 \mathrm{~A} 2$ fracture, due to an inadequate varus reduction, had a cutout that required surgical revision. Another case of type 31A2 fracture evolved to avascular necrosis of the femoral head after the fracture healed. Finally, one case of type 3143 fracture, due to inadequate reduction in the sagittal plane, evolved to delayed healing, and the nail broke 13 months after surgery. Conclusion: Osteosynthesis with proximal nail to treat unstable trochanteric fractures of the femur performed in patients older than 60 years, resulted in healing in most cases. Complications were different in the two subgroups studied. Adequate reduction of the fracture before introducing the nail is basic to achieve success in the procedure.

\section{Keywords - Hip fractures; Fracture fixation, internal/ methods; Femoral fractures; Bone nails; Fracture healing; Postoperative complications; Aged}

\section{INTRODUÇÃO}

A fratura transtrocanteriana acomete a população mais idosa do que a fratura intracapsular do colo do fêmur; são pacientes com maior incidência de osteoporose e de co-morbidades clínicas. Normalmente, o padrão da fratura é multifragmentar e instável, determinando, nos três primeiros meses após o tratamento cirúrgico, taxa de mortalidade duas vezes maior do que no grupo de fraturas do colo do fêmur ${ }^{(1)}$.

Quanto ao tratamento, a estabilização cirúrgica é a opção de escolha para a fratura trocantérica, independente do tipo ou padrão do desvio. A meta do tratamento cirúrgico é obter redução e fixação estáveis que propiciem ao paciente mobilização ativa e passiva precoces. Uma vez que no idoso a deambulação sem carga sobre o membro fraturado nem sempre é possível, pelas condições preexistentes, a estabilização da fratura deve ser suficiente para permitir certa carga tolerada pelo paciente. Atingir este objetivo depende de 
fatores como: padrão da fratura, estabilidade da redução, qualidade do osso e método de fixação interna ${ }^{(2)}$.

Desde que o tratamento cirúrgico foi estabelecido como a única maneira de evitar as complicações advindas do confinamento ao leito do paciente idoso, que apresenta fratura trocantérica do fêmur, a melhor opção de fixação dessa modalidade de lesão tem sido o foco de discussão. Vários implantes foram desenvolvidos no intuito de se conseguir uma estabilização adequada, principalmente nos casos em que existe instabilidade do foco de fratura ${ }^{(3-8)}$.

Simmermacher et al descreveram o resultado de estudo multicêntrico com avaliação da haste proximal do fêmur, $\mathrm{PFN}^{\circledR}$-AO/ASIF, que apresenta a proposta de ser um implante intramedular associado a um parafuso deslizante de fixação na cabeça do fêmur, com um segundo parafuso de menor diâmetro para aumentar a estabilidade rotatória desta fixação cefálica, além de possuir desenho em que a parte distal da haste tornava-se mais afilada para minimizar o risco de fratura abaixo da ponta do implante( ${ }^{(9)}$.

O objetivo desta pesquisa é identificar a taxa de consolidação da fratura trocantérica instável submetida a osteossíntese com a haste proximal femoral ( $\mathrm{PFN}^{\circledR}-\mathrm{AO} /$ ASIF), por meio do estudo de grupo de 45 pacientes com idade igual ou superior a 60 anos.

\section{MÉTODOS}

No período de novembro de 1999 a março de 2004, 397 fraturas proximais do fêmur foram operadas no Instituto Nacional de Traumatologia e Ortopedia do Ministério da Saúde (INTO-MS). Deste grupo, 45 pacientes com idades entre 60 e 93 anos, que apresentavam fraturas trocantéricas instáveis do fêmur, foram submetidos a osteossíntese com $\mathrm{PFN}^{\circledR}$ curto. A fratura trocantérica foi definida como aquela que compromete a região desde a parte extracapsular do colo femoral até a área do pequeno trocanter e grande trocanter.

Os critérios de inclusão para este estudo foram: 1) fraturas trocanterianas do fêmur instáveis, classificadas como tipo $31 \mathrm{~A} 2$ ou $31 \mathrm{~A} 3$; 2) paciente com idade maior do que 60 anos no momento da fratura; 3 ) habilidade para deambular antes da fratura.
Os critérios de exclusão para este estudo foram: 1) fraturas com traço intra-articular ou basocervical pura; 2) fraturas com traço apresentando extensão subtrocanteriana; 3) pacientes em que a fixação da fratura foi efetuada com haste longa do $\mathrm{PFN}^{\circledR}$; 4) fraturas associadas em outros segmentos ósseos; 5) fraturas patológicas por lesões tumorais.

O sexo feminino predominou com 37 casos $(82,2 \%)$ e apenas oito pacientes eram do sexo masculino $(17,7 \%)$. Com relação ao lado comprometido, houve equilíbrio entre 23 casos do lado direito e 22 pacientes com fratura do lado esquerdo. A faixa etária variou entre 60 e 93 anos de idade, sendo a média da idade de 77,1 anos. A média de dias entre a data do trauma que originou a fratura e o dia da cirurgia foi de oito dias, com quatro dias de média entre a internação no hospital e a data do procedimento cirúrgico. Quanto à causa da fratura: 42 pacientes foram vítimas de queda ao solo $(93,3 \%)$, enquanto três sofreram trauma de maior energia, sendo que dois foram atropelamento em via pública $(4,4 \%)$ e um sofreu queda de cerca de $2 \mathrm{~m}$ de altura $(2,2 \%)$. Todos os pacientes apresentavam fratura trocantérica isolada do fêmur, sem outras lesões do sistema músculo-esquelético.

As fraturas foram divididas de acordo com a classificação $\mathrm{AO} / \mathrm{OTA}^{(10)}$. Todas apresentavam padrão instável, sendo 22 fraturas do tipo $31 \mathrm{~A} 2(48,8 \%)$ e 23 do tipo 31A3 (51,1\%) (tabela 1).

TABELA 1

Freqüência quanto ao tipo de fratura

\begin{tabular}{ccr}
\hline Tipo de fratura & Freqüência & \multicolumn{1}{c}{$\%$} \\
\hline 31A2 & 22 & 48,89 \\
31A3 & 23 & 51,11 \\
Total & 45 & 100,00 \\
\hline
\end{tabular}

Fonte: INTO-MS (1999-2004).

A qualidade óssea foi avaliada pelo índice de $\operatorname{Singh}^{(1)}$, constatado no momento da internação, por meio do estudo radiográfico, incidência panorâmica da bacia, em que $82,22 \%$ dos pacientes apresentavam índice menor ou igual a IV, caracterizando perda óssea considerável (tabela 2). 
TABELA 2

Índice de Singh da extremidade proximal do fêmur nos pacientes operados

\begin{tabular}{ccr}
\hline Índice de Singh & Freqüência & $\%$ \\
\hline II & 23 & 51,11 \\
III & 14 & 31,11 \\
IV & 8 & 17,78 \\
Total & 45 & 100,00 \\
\hline
\end{tabular}

Fonte: INTO-MS (1999-2004).

Todos os pacientes foram submetidos a osteossíntese com a haste curta $\mathrm{PFN}^{\circledR}-\mathrm{AO} / \mathrm{ASIF}^{(9)}$, tendo sido utilizada de rotina a redução indireta da fratura em mesa ortopédica com auxílio da radioscopia. A escolha da haste foi feita após planejamento pré-operatório, de acordo com o ângulo cervicodiafisário da extremidade proximal do fêmur contralateral, tendo variado entre $125^{\circ}, 130^{\circ}$ e $135^{\circ}$ de inclinação entre os parafusos do colo e o eixo da haste intramedular. Em todos os casos a haste intramedular era do tipo curta, com diâmetro de $17 \mathrm{~mm}$ proximal e $10 \mathrm{~mm}$ distal. O bloqueio proximal foi efetuado sempre com o parafuso deslizante de cor rosa associado ao parafuso anti-rotatório de cor azul, sendo que a soma da distância entre a ponta do parafuso deslizante e o ápice do centro da cabeça, nas duas incidências radiográficas, foi menor ou igual a $20 \mathrm{~mm}$ na maioria dos casos. Quanto ao bloqueio distal, apenas um parafuso cortical de cor verde era colocado no orifício estático da haste.

A qualidade da redução da fratura e o posicionamento do implante foram avaliados pelo estudo radiográfico no pós-operatório imediato, pela radiografia em ântero-posterior (AP) e perfil do fêmur proximal. A redução era considerada ideal quando o ângulo cervicodiafisário na radiografia em AP encontrava-se entre 130-135'; abaixo destas medidas, foi considerada redução em varo. Na incidência em perfil, foi avaliado o eixo de alinhamento do fêmur, para se detectar uma redução inadequada no plano sagital, que se caracteriza pela deformidade em flexão do fragmento proximal, que ocorre principalmente no padrão de fratura $31 \mathrm{~A} 3$.

O posicionamento do implante era avaliado pelo chamado índice de Baumgaertner (tip apex distance) ${ }^{(12)}$, que é o somatório das distâncias entre a ponta do parafuso deslizante e o centro da cabeça femoral, nas duas incidências radiográficas citadas anteriormente.

O tempo médio de utilização da radioscopia foi de 102,4 segundos, variando de 61 a $185 \mathrm{seg}$, nas 45 fraturas trocantéricas instáveis, sendo que não existiu diferença significativa entre o tempo de radioscopia utilizado para a redução e fixação com o implante $\mathrm{PFN}^{\circledR}$ $\mathrm{AO} / \mathrm{ASIF}$ entre os dois grupos de fratura (31A2 e 31A3) (tabela 3).

TABELA 3

Relação entre o tipo de fratura, o tempo médio de radioscopia durante a cirurgia

\begin{tabular}{lccc}
\hline Tipo de fratura & 31A2 & 31A3 & p \\
\hline $\begin{array}{l}\text { Tempo de } \\
\text { radioscopia } \\
\text { (segundos) }\end{array}$ & $106,9 \mathrm{seg}$ & $98,1 \mathrm{seg}$ & $\begin{array}{c}0,62 \\
\text { (não significativo) }\end{array}$
\end{tabular}

Fonte: INTO-MS (1999-2004).

A deambulação no segundo dia de pós-operatório foi estimulada em todos os casos, com o paciente sendo assistido por um profissional da medicina física para que iniciasse a marcha com auxílio de andador e sentasse fora do leito, sempre sob supervisão e respeitando-se o quadro álgico do paciente. Apenas seis pacientes $(13,3 \%)$, que apresentavam complicações clínicas imediatas no pós-operatório, não conseguiram cumprir este protocolo, porém, na primeira revisão ambulatorial com quatro semanas, três destes já conseguiam deambular com andador e os demais utilizavam cadeiras de rodas.

O presente trabalho é um estudo de coorte prospectivo com análises transversais, sobre a fratura trocantérica instável do fêmur, tratada cirurgicamente por meio de estabilização com haste intramedular. Os pacientes foram divididos em dois grupos de acordo com o padrão da fratura, porém com variáveis basais, tais como idade, sexo, mecanismo do trauma e instabilidade. Portanto, a avaliação quanto à eficácia do método, nas fraturas instáveis trocantéricas do fêmur, foi estimada em relação à consolidação da fratura, sendo então os dois grupo avaliados em conjunto com um 
número total de 45 casos. A avaliação das complicações foi separada por grupo uma vez que cada tipo de fratura apresentou diferentes aspectos neste sentido.

\section{RESULTADOS}

A análise da perda de massa óssea medida pelo índice de Singh $^{(11)}$ e sua relação com a falha da osteossíntese, o chamado cutout, não existiu relação identificável entre este dois fatores, pois em apenas um caso do tipo 31A2 ocorreu esta complicação (tabela 4).

TABELA 4

Relação entre o índice de Singh da extremidade proximal do fêmur do paciente e a incidência de cutout no pós-operatório

\begin{tabular}{cl}
\hline Índice de Singh & Cutout \\
\hline II (23) & Nenhum \\
III (14) & 1 caso \\
IV (8) & Nenhum \\
\hline
\end{tabular}

Fonte: INTO-MS (1999-2004).

A qualidade da redução no pós-operatório foi considerada ideal em 36 casos $(80,0 \%)$, em cinco casos o ângulo cervicodiafisário na radiografia em AP era menor que $130^{\circ}$, caracterizando a redução em varo, sendo que todas estas eram fraturas do tipo 31A2. Em quatro casos de fratura do tipo $31 \mathrm{~A} 3$ as radiografias em perfil evidenciaram deformidade em flexão do fragmento proximal do fêmur (tabela 5).

\section{TABELA 5}

Avaliação da qualidade da redução pós-operatória através do ângulo cervicodiafisário e da deformidade em flexão

\begin{tabular}{|c|c|c|}
\hline Tipo de fratura & $\begin{array}{c}\text { Ângulo } \\
\text { cervicodiafisário } \\
\text { (RX em AP) }\end{array}$ & $\begin{array}{l}\text { Deformidade } \\
\text { em flexão } \\
\text { (RX em perfil) }\end{array}$ \\
\hline $31 \mathrm{~A} 2$ & $<130^{\circ}=5$ casos & Ausente \\
\hline $31 \mathrm{A3}$ & $<130^{\circ}=$ Ausente & Presente em 4 casos \\
\hline
\end{tabular}

Fonte: INTO-MS (1999-2004).

Quanto à qualidade do posicionamento do implante, o TAD foi maior que $20 \mathrm{~mm}$ em cinco casos de fratura $31 \mathrm{~A} 2$, os quais eram os mesmos em que a redução foi considerada em varo pelo ângulo cervicodiafisário menor que $130^{\circ}$ (tabela 6).

TABELA 6

Distância entre a ponta do parafuso deslizante e o ápice da cabeça do fêmur

\begin{tabular}{ccr}
\hline Distância ponta-ápice & Freqüência & \multicolumn{1}{c}{$\%$} \\
\hline$<20 \mathrm{~mm}$ & 40 & 88,89 \\
$>20 \mathrm{~mm}$ & 5 & 11,11 \\
Total & 45 & 100,00 \\
\hline
\end{tabular}

Fonte: INTO-MS (1999-2004).

Em sete pacientes foi necessária a realização de novo procedimento $(15,5 \%)$, sendo que em quatro destes casos o segundo procedimento cirúrgico foi decorrente da necessidade da retirada do material de síntese. Nestes casos, a redução inicial da fratura era inadequada, com o ângulo cervicodiafisário menor que $130^{\circ}$, o que acarretou, durante a evolução, a migração dos parafusos cefálicos, motivando a retirada do implante com a fratura consolidada em varo. Em dois destes casos houve o chamado efeito " $Z$ ”, descrito por Werner-Tutschku et al ${ }^{(13)}$, complicação típica deste tipo de implante, em que ocorre migração em sentidos contrários do dois parafusos do bloqueio proximal implantados na região cefálica do fêmur. Nos outros dois casos, a migração dos dois parafusos proximais ocorreu na mesma direção com impacção do foco de fratura e conseqüente consolidação óssea, porém devido ao incômodo causado no paciente os parafusos proximais foram retirados.

Em um caso, em que a redução inicial restou em varo, ocorreu o chamado cutout, que é a migração do parafuso anti-rotatório em direção superior na cabeça do fêmur, com extrusão e perda da fixação cefálica devido à reabsorção em torno do parafuso deslizante, com aumento da deformidade e consolidação final com ângulo cervicodiafisário em torno de $100^{\circ}$.

Outro caso necessitou de segundo procedimento: tratou-se de paciente que evoluiu para a consolidação da fratura no quarto mês de pós-operatório, porém no décimo-sexto mês apresentou dor progressiva no quadril operado, tendo sido diagnosticada necrose avascular da cabeça do fêmur. A paciente foi submetida à artroplastia total do quadril híbrida, com componente 
acetabular sem cimento e cimentação do componente femoral.

Em quatro casos no grupo das fraturas 31A3 após a colocação da haste intramedular, ocorreu deformidade em flexão e rotação lateral do fragmento proximal, sendo que em três casos a deformidade era pequena, existindo cerca de $50 \%$ de contato entre os fragmentos na incidência radiográfica lateral, o que não causou problemas para a consolidação óssea. Em um único caso, em que esta deformidade era mais acentuada, a consolidação não ocorreu, sendo que a haste intramedular manteve a redução por 13 meses, quando então fraturou por fadiga do metal devido a falta de consolidação. A paciente foi reoperada com retirada do implante quebrado, fresagem do canal, colocação de haste longa com bloqueio cefálico e distal, com correção da deformidade em flexão do fragmento proximal e enxertia óssea com osso ilíaco. A consolidação óssea ocorreu após três meses da segunda cirurgia.

Pelo exposto, 44 casos evoluíram para a consolidação da fratura, com média de 3,2 meses, variando entre dois e sete meses. Um caso do tipo $31 \mathrm{~A} 3$ evolui para retarde de consolidação, com conseqüente quebra do implante por fadiga e em cinco fraturas do tipo 31A2 a consolidação ocorreu com deformidade em varo da extremidade proximal do fêmur.

As complicações clínicas inerentes ao tipo de paciente estudado, tais como: trombose venosa profunda, infecção respiratória, alterações cardiológicas, infecção urinária e outras, não foram avaliadas especificamente, uma vez que o objetivo foi analisar a consolidação óssea e as possíveis complicações ortopédicas com o método de fixação. Estas complicações clínicas ocorreram em uma proporção similar a outros métodos de tratamento desta lesão, sem causar interferência nos resultados, sendo que todos os pacientes foram acompanhados por um período mínimo de seis meses de pós-operatório, sem existir nenhum óbito nesse período.

\section{DISCUSSÃO}

Davis et al demonstraram que nas fraturas trocantéricas instáveis, tipos 31A2 e 31A3, o sistema de fixação interna e seu perfeito posicionamento na extremidade proximal do fêmur influem diretamente no resultado final, pois a falta de estabilização dos fragmentos fraturários, invariavelmente, acarreta falha do implante e/ou falha da consolidação óssea ${ }^{(14)}$.

Parker et al, após avaliação por metanálise, comparando as hastes cefalomedulares com os implantes extramedulares empregados no tratamento da fratura extracapsular proximal do fêmur, concluíram que o emprego do parafuso deslizante associado à placa-tubo, constitui-se na grande opção de tratamento dessas fraturas, principalmente no tipo $31 \mathrm{~A} 2$, em que o traço de fratura propicia excelente função para o sistema dinâmico de deslizamento do parafuso cervical ${ }^{(15)}$. Porém, no tipo 31A3, com padrão reverso de fratura, e na fratura subtrocantérica isso não ocorre. Para estes casos, as hastes intramedulares estariam melhor indicadas, porém concluem que mais estudos prospectivos eram necessários para confirmar esta suposição ${ }^{(15)}$. Adams et al realizaram estudo prospectivo randomizado comparando o emprego dos dois sistemas de fixação da fratura trocantérica e observaram que o uso da haste cefalomedular está associado a maior risco de complicações pós-operatórias. Concluíram, dessa forma, que o emprego rotineiro das hastes não deve ser recomendado e que o procedimento padrão para a fixação da fratura trocantérica é a placa e o parafuso deslizante. A crítica a estes estudos refere-se a que em realidade a amostra analisada é composta de fraturas trocantéricas estáveis e instáveis, sendo que não existe separação entre os diversos tipos, o que pode alterar os resultados ${ }^{(16)}$.

Baumgaertner et al, Hardy et al e Schipper et al realizaram estudos randomizados semelhantes aos anteriores comparando os resultados de dois métodos ${ }^{17-}$ 19). Quando as fraturas estáveis e instáveis eram avaliadas separadamente, encontraram diferenças significativas nos padrões analisados. A haste intramedular está associada a um tempo cirúrgico menor, a menor sangramento peroperatório, e a capacidade de deambulação precoce no pós-operatório ${ }^{(17-19)}$. Nós utilizamos o critério de instabilidade para poder avaliar o desempenho das hastes cefalomedulares em um gru- 
po de fraturas que apresentavam risco maior de complicações.

Com relação ao tempo de exposição à radioscopia para a execução do procedimento, Dousa et al, estudando 41 casos de fraturas fixadas com $\mathrm{PFN}^{\circledR}$, apresentaram média de 2,9 min (de 1 a 6 min) ${ }^{(20)}$. Nossos achados são bastante inferiores com média de 1,7 min (de 1 a $3 \mathrm{~min}$ ); quando comparamos separadamente o grupo de fraturas 31A2 (1,7 min) e o grupo 31A3 (1,6 min), não existiu diferença significativa, o que sugere que o tipo de instabilidade não acarretou dificuldades durante a redução da fratura e sua fixação.

Outro achado importante do nosso estudo diz respeito ao padrão de osteoporose apresentado pelo paciente antes da fratura. O índice de Singh utilizado para esta avaliação mostrou que apenas oito pacientes $(17,7 \%)$ não apresentavam sinais radiológicos de perda de massa óssea. Os $37(82,2 \%)$ casos restantes foram classificados como índices II e III. Todavia, apenas um caso com índice III evoluiu para cutout, com falha da fixação $(2,2 \%)$. O que nos sugere que a qualidade da redução da fratura e a colocação adequada do implante são mais importantes do que a qualidade óssea.

As principais complicações encontradas em nosso estudo estão em acordo com as publicações anteriores sobre este tipo de implante, as quais revelam taxa de cutout variando entre 0,7 e 10,6\%, e de fratura da diáfise abaixo da ponta da haste entre 0 a 2,1\%(20-27). Em nossa casuística encontramos apenas um caso de $\mathrm{Cu}$ tout $(2,2 \%)$ e nenhum caso de fratura do implante.

A colocação do implante obedecendo o conceito introduzido por Baumgaertner et al, em que a distância entre a ponta do parafuso deslizante e o centro da cabeça do fêmur não deve ser maior que $10 \mathrm{~mm}$ na incidência em AP e no perfil, determina o perfeito posicionamento do parafuso deslizante facilitando a telescopagem do sistema dinâmico do implante, minimizando o risco de cutout $^{(12)}$. Porém, o $\mathrm{PFN}^{\circledR}$, por possuir dois parafusos para fixação proximal, acarreta certa dificuldade para a perfeita posição no centro da cabeça do fêmur, o que é mais fácil de ser obtido quando se utiliza um parafuso deslizante e a placa-tubo. Dessa forma, existe maior tendência ao posicionamento do pa- rafuso deslizante em localização mais inferior na imagem radiográfica na incidência ântero-posterior, sobretudo, em mulheres com colo e cabeça do fêmur curto. Todavia, o conceito deve sempre nortear o cirurgião, lembrando que no perfil não há dificuldade para alcançar tal objetivo, e que mesmo que o parafuso deslizante fique localizado em posição mais baixa na cabeça do fêmur, a distância da ponta do implante ao osso subcondral nunca deve ultrapassar $10 \mathrm{~mm}$, tanto na incidência ântero-posterior quanto na visão lateral da cabeça do fêmur ${ }^{(28-30)}$.

O fenômeno cutout decorre, basicamente, da redução inicial da fratura em varo e da conseqüente colocação incorreta do implante na extremidade proximal do fêmur; portanto, a redução deve reconstituir o ângulo cervicodiafisário normal de $130^{\circ}-135^{\circ}$ para que o implante possa ser perfeitamente posicionado ${ }^{(31)}$. Nos nossos casos em que houve falha da osteossíntese, a redução inicial da fratura era inadequada, sendo considerado erro técnico, o qual precisa ser evitado no tratamento da fratura trocantérica do fêmur, seja qual for o implante utilizado. Este tipo de complicação ocorreu principalmente nos casos de fratura do tipo $31 \mathrm{~A} 2$. Observamos que em cinco a redução inicial era insatisfatória e o TAD era maior do que $20 \mathrm{~mm}$, sendo que em um destes casos ocorreu o chamado cutout, ou seja, a ruptura da cortical óssea por um dos parafusos de bloqueio cefálico, determinando dor e limitação funcional ao paciente, levando a retirada do implante, com a fratura evoluindo para a consolidação em varo (figuras 1,2 e 3). Nos outros quatro casos em que existia redução em varo, ocorreu a migração dos parafusos de bloqueio proximais. Em dois destes, ocorreu o chamado efeito ' $Z$ ', que se trata da migração em sentido contrário dos dois parafusos, o que também determinou a retirada dos implantes. Este tipo de complicação é exclusivo de implantes que possuem a fixação proximal com dois parafusos na região cefálica do fêmur. No caso do $\mathrm{PFN}^{\circledR}$, o diâmetro diferente entre os parafusos deslizante e o anti-rotatório, favorece este tipo de desvio em sentido oposto. A utilização do parafuso anti-rotatório na fixação proximal deste tipo de haste intramedular não parece aumentar a estabilidade do sistema, pois pensamos que a qualidade da re- 


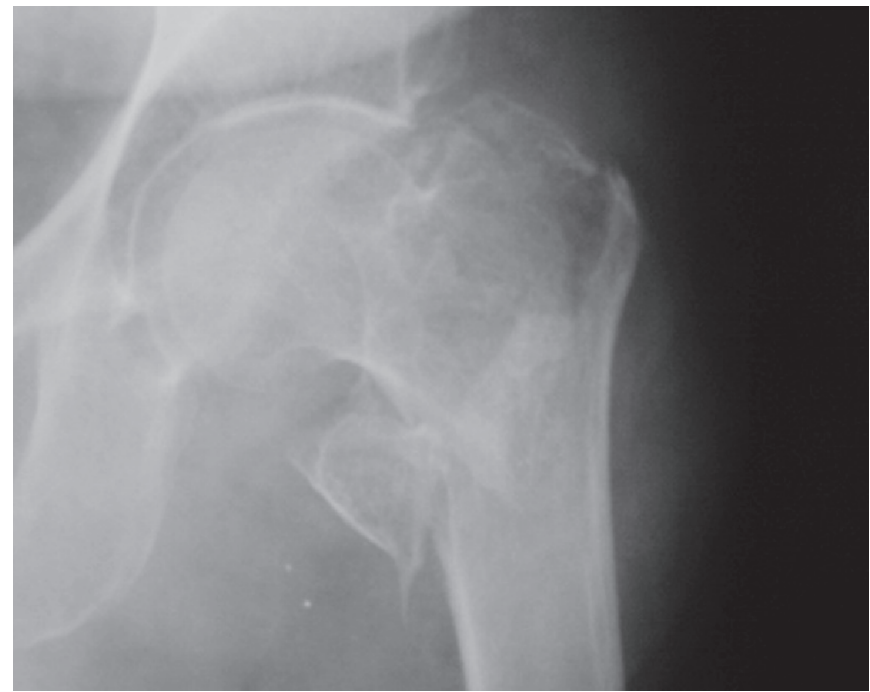

Figura 1 - Caso no 12 . Radiografia pré-operatória na incidência em AP evidenciando fratura trocantérica instável tipo 31A2.

Figura 2 - Caso $\mathrm{n}^{\circ}$ 25. Radiografia pós-operatória na incidência em AP, com dois meses de evolução, evidenciando redução insuficiente com desvio residual em varo, com extrusão do parafuso deslizante, com conseqüente evolução para a complicação conhecida como cutout.

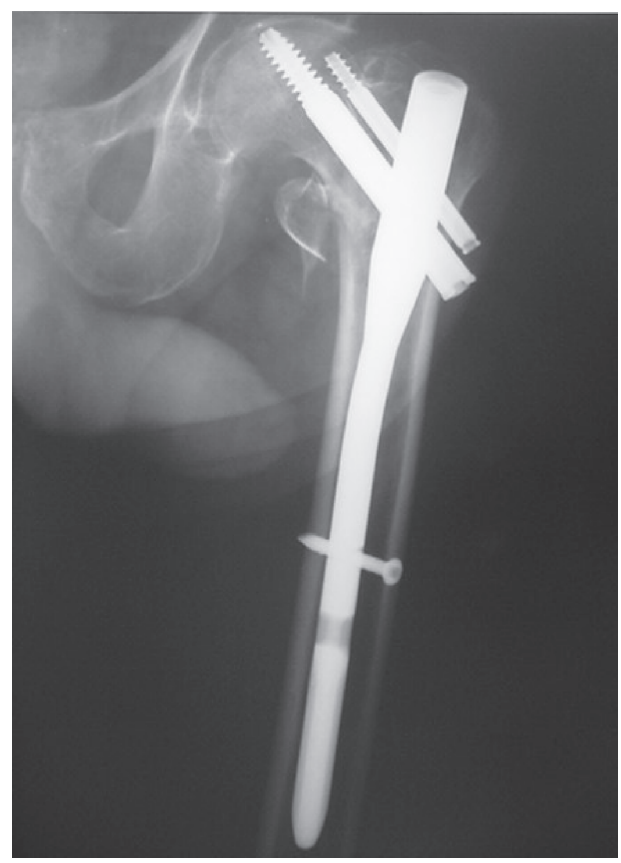

dução é o fator mais importante para prevenir as complicações estudadas (figuras 4 e 5).

Werner-Tutschku et al concordam que a principal razão para a migração dos parafusos proximais e o cutout é a redução inicial insatisfatória, geralmente em varo, sendo que os autores recomendam que ângulo cervicodiafisário menor que $125^{\circ}$ não deve ser acei-

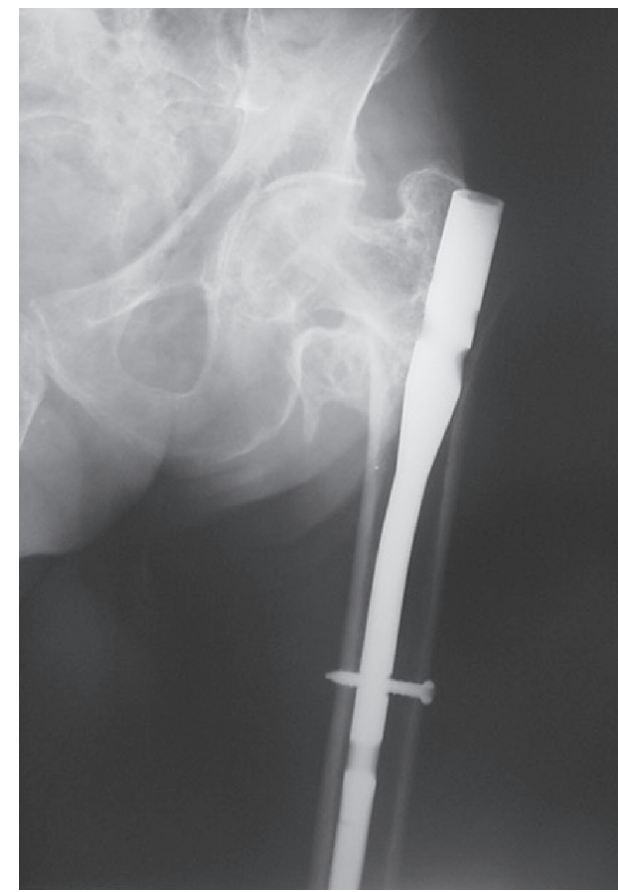

Figura 3 - Caso $\mathrm{n}^{\circ}$ 25. Radiografia pós-operatória na incidência em $A P$, com quatro meses de evolução, após reoperação com retirada dos parafusos proximais, com consolidação em varo da fratura, complicação típica do tipo de fratura 31A2.

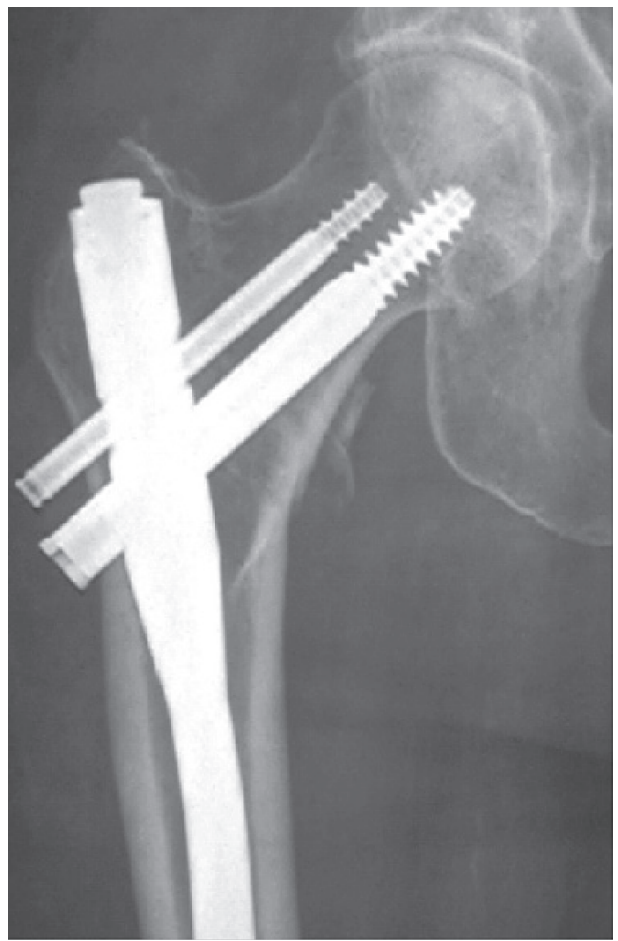

Figura 4-Caso $\mathrm{n}^{\circ}$ 21. Radiografia pós-operatória na incidência em $\mathrm{AP}$, com dois meses de evolução, evidenciando redução excelente, porém a escolha dos parafusos proximais foi inadequada, com distância da ponta do parafuso deslizante ao ápice da cabeça do fêmur maior que $10 \mathrm{~mm}$.

to ${ }^{(13)}$. Windolf et al frisam, ainda, que a principal razão para a falência da fixação é a redução inadequada e a escolha incorreta do tamanho dos parafusos proxi- 


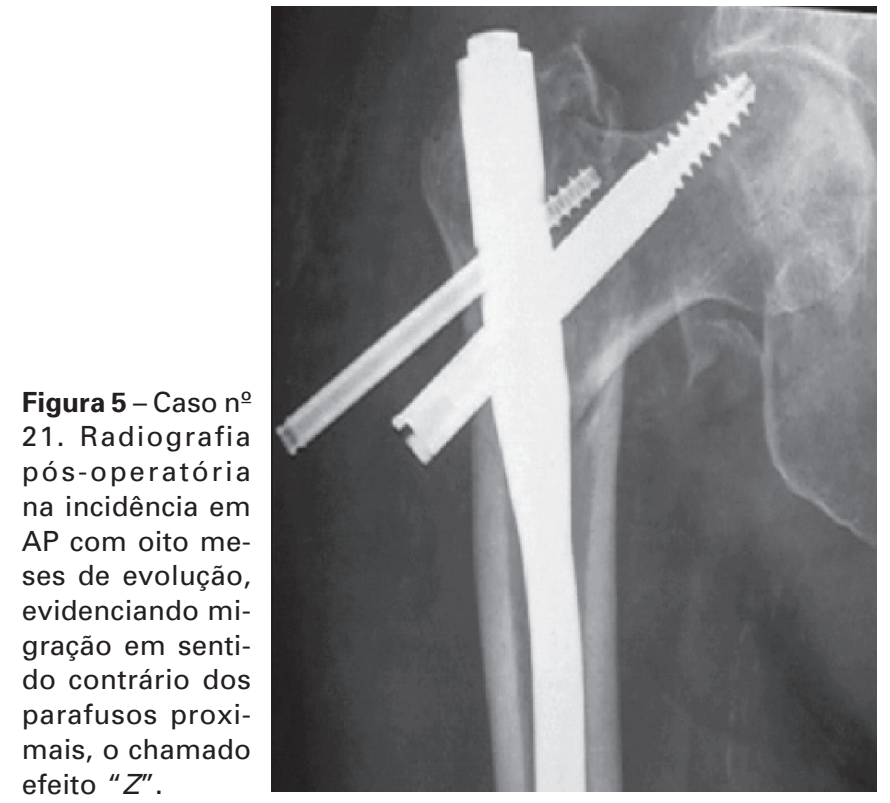

mais ${ }^{(31)}$. Nossa casuística também reforça esta teoria, de que a qualidade da redução da fratura antes da colocação do implante é fundamental para evitar as complicações detectadas neste estudo.

Nas fraturas do tipo 31A3, que se comportam com padrão similar às fraturas subtrocantéricas do fêmur, a complicação mais freqüiente foi a deformidade em flexão e rotação externa do fragmento proximal. Isto ocorreu em quatro dos 22 casos analisados de fratura trocantérica de traço reverso $(18,1 \%)$. Nesse aspecto, julgamos que a presença de um sinal de risco por esta complicação é o tipo de traço de fratura visto na incidência radiográfica em perfil, ou seja, no plano sagital. Esta deformidade ocorre quando a fratura se inicia posterior no trocanter maior e segue em direção anterior, acarretando um típico esporão que, normalmente, por ação da musculatura flexora e abdutora do quadril, determina flexão e rotação externa do segmento proximal do fêmur. Nessa situação, caso a introdução da haste não seja efetuada de maneira adequada, ou seja, mais anterior, existe o risco deste pequeno fragmento de cortical posterior do grande trocanter ser destruído por ocasião da fresagem, ou mesmo a haste criar falso trajeto e entrar diretamente no segmento diafisário sem ter controle sobre a flexão do fragmento proximal. O conceito de fixação desta haste proximal é cefalodiafisária, ou seja, a ancoragem do implante é feita no núcleo duro da cabeça do fêmur e na região da diáfise. Sendo assim, se conseguirmos essa adequada fixação cefálica, a haste proporciona fixação estável, mesmo persistindo a deformidade em flexão do fragmento proximal, o que ocorreu em três casos de fratura $31 \mathrm{~A} 3$, sendo que apenas em um caso a fratura evoluiu para pseudartrose com quebra do implante; nos demais casos, em que existia cerca de $50 \%$ de contato entre os fragmentos, a consolidação óssea ocorreu, apesar da deformidade vista na incidência radiográfica de perfil da extremidade proximal do fêmur (figuras 6, 7 e 8). Boldin et al, analisando 55 casos submetidos à osteossíntese com PFN ${ }^{\circledR}$, propõem a abertura do foco de fratura e a utilização de cerclagem óssea para garantir a redução quando existe este tipo de deformidade ${ }^{(32)}$.

O único caso que evolui para necrose asséptica da cabeça do fêmur necessitou de reoperação. Acreditamos que esta complicação não parece estar relaciona-

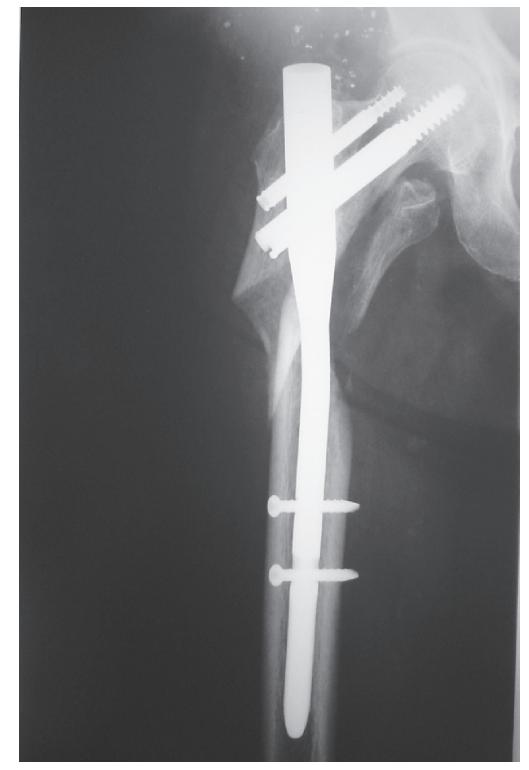

Figura 6 - Caso no 42. Radiografias pós-operatórias na incidência em AP e perfil, com quatro meses de evolução, evidenciando redução insatisfatória com típica deformidade em flexão e rotação externa do fragmento proximal do fêmur que ocorre no tipo 31A3; resultado clínico satisfatório, com paciente deambulando com apoio de muletas, sem queixas álgicas.

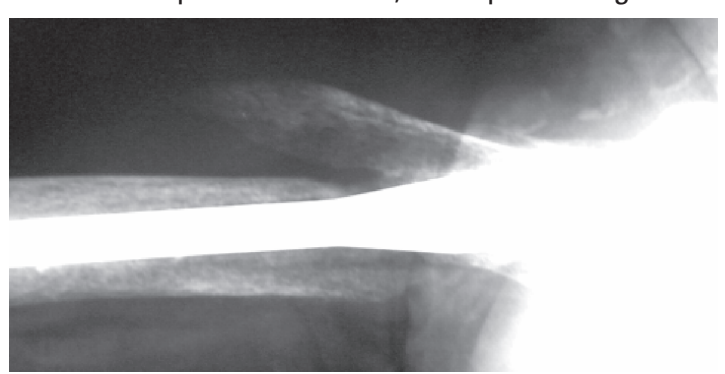


Figura 7 - Caso $\mathrm{n}^{\mathrm{0}}$ 7. Radiografia pós-operatória na incidência em AP e perfil, com 13 meses de evolução, evidenciando fratura por fadiga do implante, na altura do primeiro orifício de bloqueio distal da haste intramedular.

Figura 8-Caso no 42. Radiografia pós-operatória na incidência em AP mostrando a conversão da haste curta quebrada para uma fixação com haste longa, que evolui para a consolidação aos 18 meses após a primeira cirurgia.
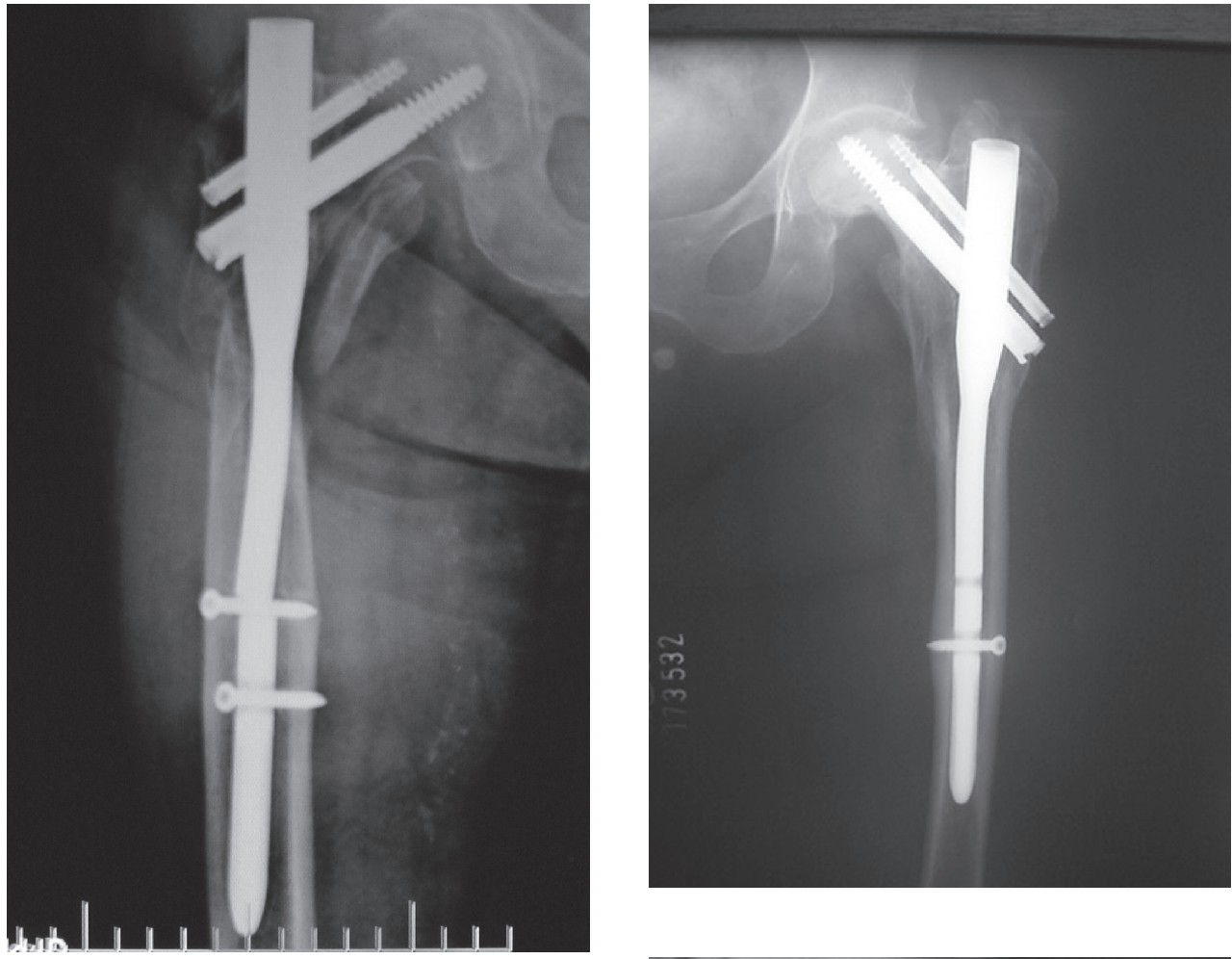

Figura 9 - Caso $\mathrm{n}^{\mathrm{o}}$ 9. Radiografia pós-operatória na incidência em AP com nove meses de evolução, evidenciando fratura consolidada, com sinais radiológicos evidentes de necrose avascular da cabeça do fêmur.

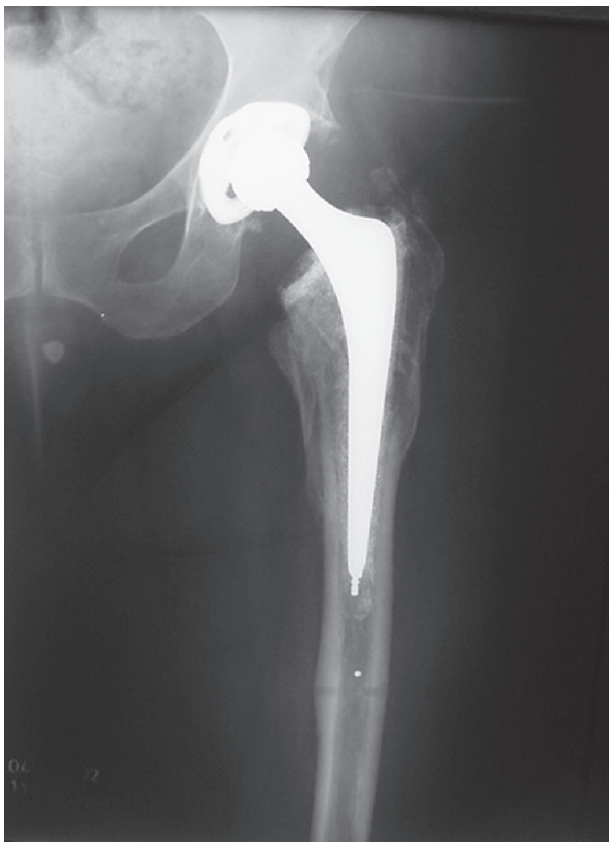

Figura 10 - Caso no 9. Radiografia pós-operatória na incidência em AP com 12 meses de evolução, após reoperação com realização de artroplastia total híbrida do quadril.

do ao tipo de implante utilizado, pois o ponto de entrada do $\mathrm{PFN}^{\circledR}$ é a ponta do grande trocanter, região que não coloca em risco a vascularização da cabeça do fêmur; além disso, existem alguns relatos de casos de necrose avascular da cabeça do fêmur associada a 
fraturas trocantéricas que foram submetidas a outros tipos de implantes, inclusive com placa-tubo tipo DHS ${ }^{\circledR}$ e DCS ${ }^{\circledR}$, o que sugere que esta complicação deve resultar do trauma inicial que determinou a fratura ${ }^{(33-36)}$ (figuras 9 e 10).

Autores são de opinião que a haste $\mathrm{PFN}^{\circledR}$ possibilita a obtenção de excelentes resultados no tratamento da fratura trocantérica instável do fêmur, principalmente quanto à estabilidade propiciada pelo implante, que permite deambulação precoce com melhor reabilitação funcional do paciente idoso e alto índice de consolidação decorrente da preservação do envelope de partes moles em torno da fratura ${ }^{(37-40)}$.

\section{CONCLUSÃO}

A osteossíntese com haste proximal femoral $\mathrm{PFN}^{\circledR}$ na fratura trocantérica instável do fêmur, em pacientes com idade superior a 60 anos, resultou na consolidação da maioria dos casos.

As complicações foram distintas no dois subgrupos estudados, a saber, consolidação viciosa em varo nas fraturas do tipo 31A2 e desvio em flexão do fragmento proximal nas do tipo $31 \mathrm{~A} 3$.

\section{REFERÊNCIAS}

1. Zuckerman JD, Skovron ML, Koval KJ, Aharonoff G, Frankel VH. Postoperative complications and mortality associated with operative delay in older patients who have a fracture of the hip. J Bone Joint Surg Am. 1995;77(10):1551-5.

2. Kyle RF. Fractures of the proximal part of the femur. J Bone Joint Surg Am. 1994;76(6):924-50.

3. Chang WS, Zuckerman JD, Kummer FJ, Frankel VH. Biomechanical evaluation of anatomic reduction versus medial displacement osteotomy in unstable intertrochanteric fractures. Clin Orthop Relat Res. 1987;(225):141-6.

4. Kyle RF, Gustilo RB, Premer RF. Analysis of six hundred and twenty-two intertrochanteric hip fractures. J Bone Joint Surg Am. 1979;61(2):216-21.

5. Koval KJ, Zuckerman JD. Hip Fractures: II. Evaluation and treatment of intertrochanteric fractures. J Am Acad Orthop Surg. 1994;2(3):150-6.

6. Cobelli NJ, Sadler AH. Ender rod versus compression screw fixation of hip fractures. Clin Orthop Relat Res. 1985;(201): 123-9.

7. Tencer AF, Johnson KD, Johnston DW, Gill K. A biomechanical comparison of various methods of stabilization of subtrochanteric fractures of the femur. J Orthop Res. 1984; 2(3):297-305.

8. Kempf I, Grosse A, Taglang G, Favreul E. [Gamma nail in the treatment of closed trochanteric fractures. Results and indications apropos of 121 cases]. Rev Chir Orthop Reparatrice Appar Mot. 1993;79(1):29-40. French.

9. Simmermacher RK, Bosch AM, Van der Werken C. The AO/ ASIF-proximal femoral nail (PFN): a new device for the treatment of unstable proximal femoral fractures. Injury. 1999; 30(5):327-32.

10. Muller ME, Allgower M, Schneider R, Wllenegger H. Manual of internal fixation. 3th ed. Berlin: Springer; 1991. p. 136-7.

11. Singh M, Riggs BL, Beabout JW, Jowsey J. Femoral trabecular-pattern index for evaluation of spinal osteoporosis. Ann Intern Med. 1972;77(1):63-7.

12. Baumgaertner MR, Curtin SL, Lindskog DM, Keggi JM. The value of the tip-apex distance in predicting failure of fixation of peritrochanteric fractures of the hip. J Bone Joint Surg Am. 1995;77(7):1058-64.

13. Werner-Tutschku W, Lajtai G, Schmiedhuber G, Lang T, Pirk1 C, Orthner E. [Intra- and perioperative complications in the stabilization of per- and subtrochanteric femoral fractures by means of PFN]. Unfallchirurg. 2002;105(10):881-5. German.

14. Davis TRC, Sher JL, Horsman A, Simpson M, Porter BB, Checketts RG. Intertrochanteric femoral fractures. Mechanical failure after internal fixation. J Bone Joint Surg Br. 1990;72(1): 26-31.

15. Parker MJ, Handoll HH. Gamma and other cephalocondylic intramedullary nails versus extramedullary implants for extracapsular hip fractures. Cochrane Database Syst Rev. 2005; (4):CD000093. Review.

16. Adams CI, Robinson CM, Court-Brown CM, McQueen MM. Prospective randomized controlled trial of an intramedullary nail versus dynamic screw and plate for intertrochanteric fractures of the femur. J Orthop Trauma. 2001;15(6):394-400.

17. Baumgaertner MR, Curtin SL, Lindskog DM. Intramedullary versus extramedullary fixation for the treatment of intertrochanteric hip fractures. Clin Orthop Relat Res. 1998; (348):87-94.

18. Hardy DC, Descamps PY, Krallis P, Fabeck L, Smets P, Bertens CL, Delince PE. Use of an intramedullary hip-screw compared with a compression hip-screw with a plate for intertrochanteric femoral fractures. A prospective, randomized study of one hundred patients. J Bone Joint Surg Am. 1998;80(5):618-30.

19. Schipper IB, Marti RK, van der Werken C. Unstable trochanteric femoral fractures: extramedullary or intramedullary fixation. Review of literature. Injury. 2004; 35(2):142-51.

20. Dousa P, Bartonícek J, Jehlicka D, Skála-Rosenbaum J. [Osteosynthesis of trochanteric fractures using proximal femoral nails]. Acta Chir Orthop Traumatol Cech. 2002;69(1): 22-30. Czech. 
21. Domingo LJ, Cecilia D, Herrera A, Resines C. Trochanteric fractures treated with a proximal femoral nail. Int Orthop. 2001;25(5):298-301.

22. Waldherr-Marty P, Beldi G, Schuster AJ, Christen B. The proximal femoral nail (PFN) for pertrochanteric femur fractures in elderly patients. J Bone Joint Surg Br. 2001;83 (Suppl 2):193.

23. Banan H, Al-Sabti A, Jimulia T, Hart AJ. The treatment of unstable, extracapsular hip fractures with the AO/ASIF proximal femoral nail (PFN)--our first 60 cases. Injury. 2002; 33(5):401-5.

24. Al-yassari G, Langstaff RJ, Jones JW, Al-Lami M. The AO/ ASIF proximal femoral nail (PFN) for the treatment of unstable trochanteric femoral fracture. Injury. 2002;33(5):395-9.

25. Saudan M, Lübbeke A, Sadowski C, Riand N, Stern R, Hoffmeyer P. Pertrochanteric fractures: is there an advantage to an intramedullary nail?: a randomized, prospective study of 206 patients comparing the dynamic hip screw and proximal femoral nail. J Orthop Trauma. 2002;16(6):386-93.

26. Fogagnolo F, Kfuri M Jr, Paccola CA. Intramedullary fixation of pertrochanteric hip fractures with the short AO-ASIF proximal femoral nail. Acta Orthop Trauma Surg. 2004; 124(1):31-7.

27. Pavelka T, Kortus J, Linhart M. [Osteosynthesis of proximal femoral fractures using short proximal femoral nails]. Acta Chir Orthop Traumatol Cech. 2003;70(1):31-8. Czech.

28. Kish B, Regev A, Goren D, Shabat S, Nyska M. Complications with the use of proximal femoral nail (PFN). J Bone Joint Surg Br. 2005;87-B(Suppl 3):376.

29. Leung KS, So WS, Shen WY, Hui PW. Gamma nails and dynamic hip screws for peritrochanteric fractures. A randomised prospective study in elderly patients. J Bone Joint Surg Br. 1992;74(3):345-51.

30. Schipper IB, Steyerberg EW, Castelein RM, van der Heijden FH, den Hoed PT, Kerver AJ, van Vugt AB. Treatment of unstable trochanteric fractures. Randomised comparison of the gamma nail and the proximal femoral nail. J Bone Joint Surg Br. 2004;86(1):86-94.
31. Windolf J, Hollander DA, Hakimi M, Linhart W. Pitfalls and complications in the use of the proximal femoral nail. Langenbecks Arch Surg. 2005;390(1):59-65.

32. Boldin C, Seibert FJ, Fankhauser F, Peicha G, Grechenig W, Szyszkowitz R. The proximal femoral nail (PFN) - a minimal invasive treatment of unstable proximal femoral fractures: a prospective study of 55 patients with a follow-up of 15 months. Acta Orthop Scand. 2003;74(1):53-8.

33. Braune C, Pohlemann T, Schandelmaier P, Tscherne $H$. [Osteonecrosis of the femoral head after osteosynthesis of a proximal femur fracture with a 95 degree condylar plate]. Unfallchirurg. 1999;102(3):236-8. German.

34. Baixauli EJ, Baixauli F Jr, Baixauli F, Lozano JA. Avascular necrosis of the femoral head after intertrochanteric fractures. $J$ Orthop Trauma. 1999;13(1):9-12. Corrected and republished in: J Orthop Trauma. 1999;13(2):134-7.

35. Chen CM, Chiu FY, Lo WH. Avascular necrosis of femoral head after gamma-nailing for unstable intertrochanteric fractures. Arch Orthop Trauma Surg. 2001;121(9):505-7.

36. Vicario C, Marco F, Ortega L, Alcobendas M, Dominguez I, López-Durán L. Necrosis of the femoral head after fixation of trochanteric fractures with Gamma Locking Nail. A cause of late mechanical failure. Injury. 2003;34(2):129-34.

37. Sadowski C, Lübbeke A, Saudan M, Riand N, Stern R, Hoffmeyer P. Treatment of reverse oblique and transverse intertrochanteric fractures with use of an intramedullary nail or a 95 degrees screw-plate: a prospective, randomized study. J Bone Joint Surg Am. 2002;84-A(3):372-81.

38. Bhatti A, Power D, Qureshi S, Khan I, Tan S. 03466 A prospective trial of proximal femoral nail versus dynamic hip screw for unstable and complex intertrochanteric fractures of the femur. J Bone Joint Surg Br. 2004;86-B(Suppl 3):377.

39. Pajarinen J, Lindahl J, Michelsson O, Savolainen V, Hirvensalo E. Pertrochanteric femoral fractures treated with a dynamic hip screw or a proximal femoral nail. A randomised study comparing post-operative rehabilitation. J Bone Joint Surg Br. 2005;87(1):76-81.

40. Menezes DF, Gamulin A, Noesberger B. Is the proximal femoral nail a suitable implant for treatment of all trochanteric fractures? Clin Orthop Relat Res. 2005;439:221-7.

\section{Declaração de inexistência de conflitos de interes-} se: Não há conflito de interesse em relação à pesquisa. 